04.2;09.1

\title{
Источник мягкого рентгеновского излучения, формируемый в сверхзвуковых газовых струях аргона под действием высококонтрастных фемтосекундных лазерных импульсов релятивистской интенсивности
}

\author{
(C) М.А. Алхимова ${ }^{1}$, С.Н. Рязанцев ${ }^{1,2}$, И.Ю. Скобелев ${ }^{1,2}$, М.Д. Мищенко ${ }^{1}$, А.С. Болдарев ${ }^{3}, \mathrm{Feng}^{\mathrm{J}} \mathrm{Jie}^{4}$, \\ Lu Xin ${ }^{4}$, Li-Ming Chen ${ }^{4}$, C.A. Пикуз ${ }^{1,2}$ \\ ${ }^{1}$ Объединенный институт высоких температур РАН, Москва, Россия \\ ${ }^{2}$ Национальный исследовательский ядерный университет „МИФИ“, Москва, Россия \\ ${ }^{3}$ Институт математического моделирования РАН, Москва, Россия \\ ${ }^{4}$ Институт фиизики Китайской академии наук, Пекин, КНР \\ E-mail: MAAlkhimova@mephi.ru
}

Поступило в Редакцию 29 октября 2019г.

В окончательной редакции 17 декабря 2019г.

Принято к публикации 17 декабря 2019г.

\begin{abstract}
Исследуется возможность создания компактного источника мягкого рентгеновского излучения без сопутствующей генерации быстрых электронов. Источник формируется при взаимодействии высококонтрастных фемтосекундных лазерных импульсов релятивистской интенсивности со сверхзвуковыми газовыми струями аргона. Найдены оптимальные условия, при которых коэффициент конверсии в мягкое рентгеновское излучение диапазона $2.9-3.3 \mathrm{keV}$ достигает $8.57 \cdot 10^{-5}$.
\end{abstract}

Ключевые слова: лазерная плазма, рентгеновское излучение, газовая мишень.

DOI: 10.21883/PJTF.2020.06.49160.18090

Лазерная плазма как яркий импульсный источник рентгеновского излучения в спектральном диапазоне $0.1-50 \mathrm{keV}$ активно исследуется в последние несколько десятилетий [1-3]. Для формирования интенсивных источников жесткого рентгеновского излучения принято использовать мишени-фольги из химических элементов с высоким атомным номером $Z \sim 30-50$. В то же время для ряда практических приложений, таких как литография или рентгенография микрообъектов, необходимо создание источников более мягкого диапазона. Для этих целей наиболее подходящими оказались газовые, кластерные или пористые мишени, облучаемые лазерными импульсами с плотностью потока $I \sim 10^{16}-10^{19} \mathrm{~W} / \mathrm{cm}^{2}$. Было показано, что повышение рентгеновской эмиссии (РЭ) возможно за счет использования наноструктурированных мишеней [4]. Однако их создание является сложным многоступенчатым и, как следствие, дорогостоящим процессом. Применение существенно более технологичных кластерных мишеней также позволяет получить достаточно высокий выход РЭ, но при этом, как правило, плазма является источником не только рентгена, но и ускоренных потоков корпускулярных частиц [5]. Наличие генерации потоков быстрых заряженных частиц часто является весьма нежелательным процессом, и задача создания „чистого“ источника рентгеновского излучения, несомненно, является очень актуальной.

Настоящая работа посвящена поиску условий, оптимальных для формирования такого источника мягкого рентгеновского излучения $\left(E_{X-r a y} \sim 3 \mathrm{keV}\right)$ в плазме, об- разующейся при нагреве аргоновых импульсных газовых мишеней высококонтрастными импульсами фемтосекундного лазера с плотностью потока $10^{16}-10^{19} \mathrm{~W} / \mathrm{cm}^{2}$. Поиск оптимальных условий осуществлялся при изменении характеристик мишени, лазерного импульса и условий фокусировки, а контроль реализовался при помощи методов высокоразрешающей рентгеновской спектроскопии.

Экспериментальные исследования проводились на лазерной установке IOP $20 \mathrm{TW}$ в Институте прикладной физики Китайской академии наук [6]. Лазерная система на основе Ti:Sapphire, работающая по схеме чирпирования, формировала высококонтрастные $\left(K_{\text {las }} \sim 10^{9}\right)$ импульсы с характерной длительностью $\tau_{\text {las }} \sim 40 \mathrm{fs}$ на средней длине волны $\lambda=800 \mathrm{~nm}$ и энергией в импульсе до $E_{\text {las }} \sim 300 \mathrm{~mJ}$. Лазерный пучок диаметром $5 \mathrm{~cm}$ фокусировался при помощи внеосевого параболического зеркала $f / 3.5$ в пятно диаметром $d \sim 5 \mu \mathrm{m}$, а плотность потока на мишени достигала $I_{\text {las }} \sim 2 \cdot 10^{19} \mathrm{~W} / \mathrm{cm}^{2}$. Сверхзвуковая импульсная струя аргона формировалась на выходе из щелевого сопла MS05-10-166 с прямоугольным сечением шириной $1.2 \mathrm{~mm}$ и длиной $5 \mathrm{~mm}$. Лазерный луч фокусировался в центр газовой струи на глубине $l_{x}=0.6 \mathrm{~mm}$ параллельно короткому краю сопла (рис. $1, a)$. Положение фокусировки относительно верхнего края сопла, т.е. по вертикальной оси $z$ вдоль распространения газовой струи, варьировалось в диапазоне $L_{z}=1-4 \mathrm{~mm}$. Давление аргона на входе в сопло менялось в диапазоне $P_{\text {gas }}=2-9 \mathrm{MPa}$. 

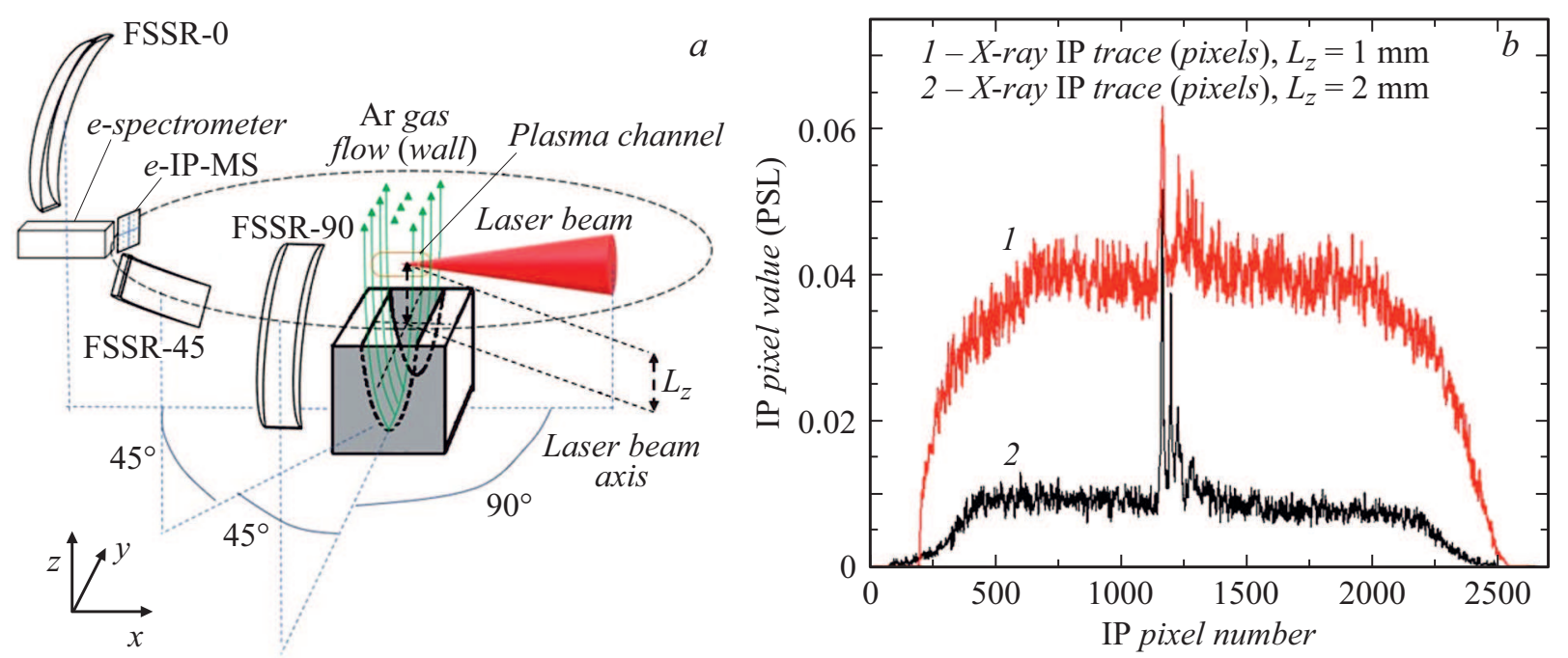

Рис. 1. Схема эксперимента $(a)$ и денситограммы спектров, зарегистрированных при различных положениях пятна фокусировки относительно края сопла $(b)$.
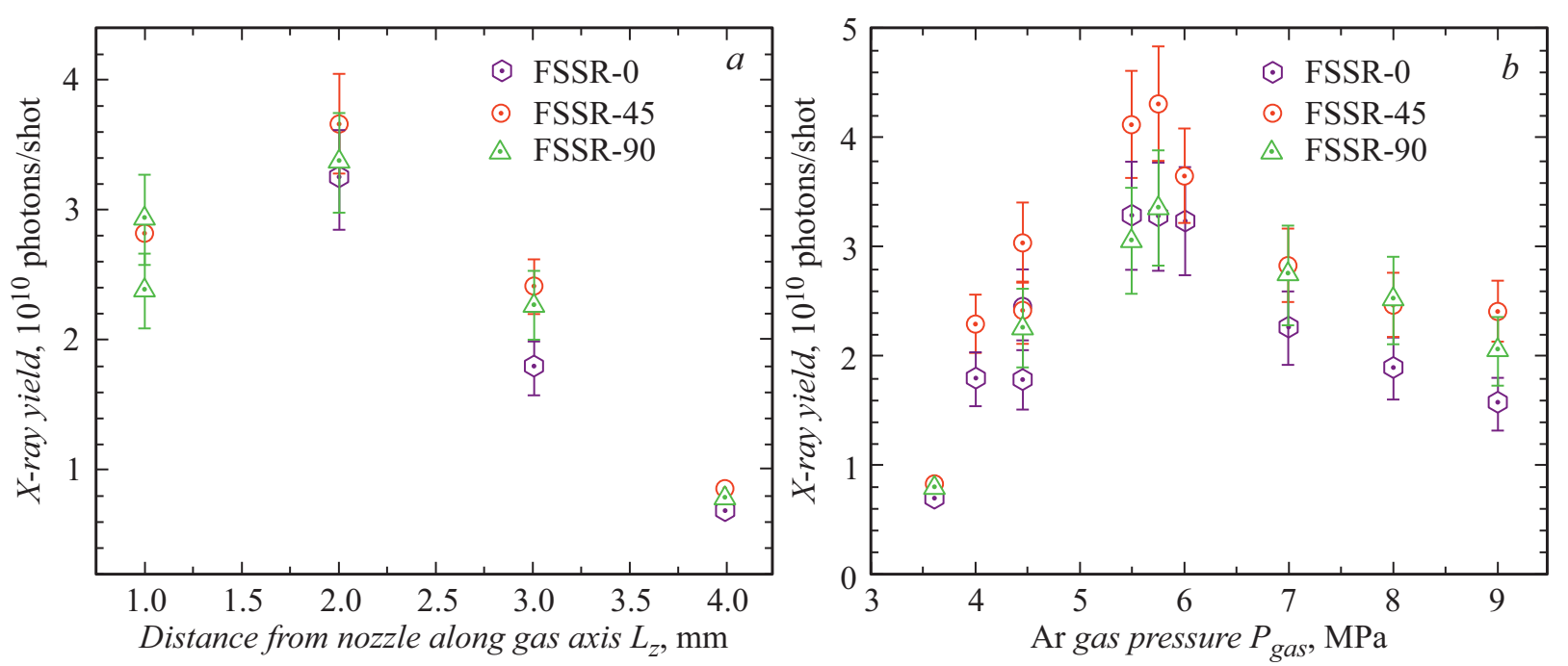

Рис. 2. Зависимости рентгеновской эмиссии от величины смещения точки фокусировки вдоль газовой струи относительно верхнего края сопла $L_{z}(a)$ и от входного давления газовой струи аргона $P_{g a s}=3.5-9 \mathrm{MPa}(b)$.

РЭ плазмы, создаваемой при взаимодействии лазерного излучения с аргоновой газовой струей, регистрировалась при помощи трех фокусирующих спектрометров с пространственным разрешением [7], оснащенных кристаллами альфа-кварца, изогнутыми по сферической поверхности с радиусом кривизны $R=150 \mathrm{~mm}$ (рис. 1,a). Спектрометры были установлены таким образом, чтобы можно было наблюдать рентгеновские эмиссионные спектры под различными углами: FSSR-0 - в направлении распространения лазерного излучения, FSSR-45 под углом $45^{\circ}$ к оси распространения лазерного пучка, FSSR-90 - перпендикулярно оси распространения лазерного пучка. Спектрометр FSSR-0, оснащенный кристаллом альфа-кварца $11 \overline{2} 0$ (межплоскостное расстояние $2 d=4.91 \AA)$, был настроен на диапазон $\lambda=3.7-4.25 \AA$, а спектрометры FSSR-45 и FSSR-90, оснащенные кристаллами альфа-кварца $11 \overline{1} 0(2 d=6.66 \AA)$, настраивались на диапазон $3.2-4.5 \AA$. Спектральное разрешение спектрометров было не хуже $\sim 3000$, а пространственное разрешение $\sim 2 \mu \mathrm{m}$. В качестве детекторов рентгеновского излучения использовались изображающие фотолюминесцентные пластины Fujifilm-TR Image Plate, защищенные от видимого излучения фильтрами из одного или двух слоев полипропилена толщиной $1 \mu \mathrm{m}$, покрытого слоем $0.2 \mu \mathrm{m} \mathrm{Al}$.

Для наблюдения спектров быстрых электронов использовался щелевой электронный спектрометр с поворотным магнитным полем $B=9000 \mathrm{G}$, для регистрации $\mathrm{keV}$-ных электронов использовались пластины Image Plate (IP), защищенные от воздействия видимого и 

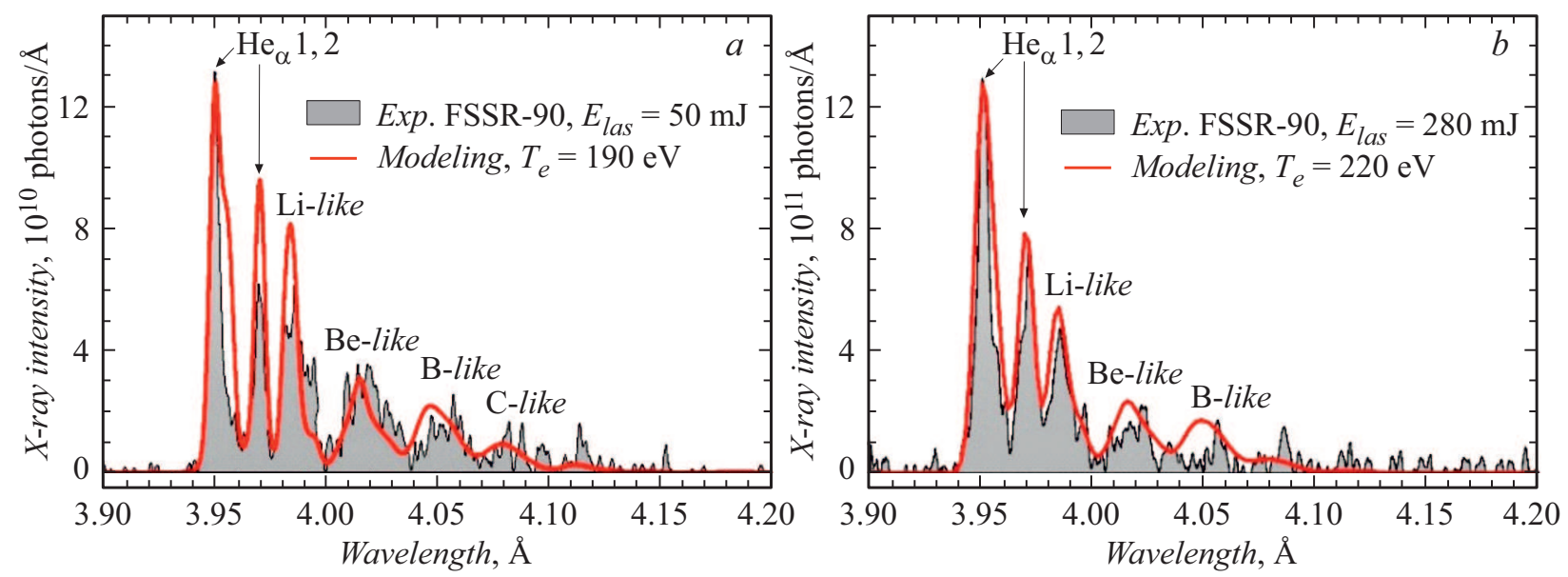

Рис. 3. Эмиссионные спектры аргоновой плазмы при энергиях лазерного импульса $E_{l a s}=50(a)$ и $280 \mathrm{~mJ}(b)$ и результаты их моделирования для ионной плотности $N_{i}=5 \cdot 10^{18} \mathrm{~cm}^{-3}$ и доли горячих $\left(T_{h o t}=3 \mathrm{keV}\right)$ электронов $0.1 \%$.

рентгеновского излучения несколькими (1-5) слоями Al-фольги толщиной $15 \mu \mathrm{m}$.

Проведенные эксперименты показали, что для создания „чистого“ рентгеновского источника наиболее критичным является выбор точки фокусировки лазерного излучения относительно верхнего края сопла. Если точка фокусировки лежит на расстояниях $L_{z}$ от края сопла, не превышающих $1 \mathrm{~mm}$, то происходит эффективная генерация быстрых электронов, и получающийся источник не является „чистым“. Это хорошо видно из денситограммы, приведенной на рис. $1, b$, где на кривой 1 отчетливо виден интенсивный квазинепрерывный спектр, обусловленный быстрыми электронами. Измеренный в этом случае щелевым спектрометром энергетический спектр быстрых электронов показал, что в плазме генерируются быстрые электроны с энергиями вплоть до $30 \mathrm{MeV}$.

Смещение точки фокусировки в положение $L_{z}=2 \mathrm{~mm}$ при сохранении остальных параметров эксперимента приводит к резкому уменьшению квазинепрерывной составляющей в зарегистрированном рентгеновском спектре (рис. $1, b$, кривая 2), что указывает на отсутствие заметного числа быстрых (MeV-ных) электронов в данном случае. Это подтверждается и отсутствием сигнала на электронном щелевом спектрометре. Изображения, зарегистрированные на IP-пластине, показывают, что ускорение электронов в данном случае происходит лишь до энергий, не превышающих $85 \mathrm{keV}$.

При еще большем удалении точки фокусировки от края сопла генерации быстрых электронов также не наблюдается, но интенсивность РЭ падает (рис. 2,a). Из рис. 2, $a$ следует, что с точки зрения получения „чистого“ источника мягкого рентгеновского излучения с характерной энергией $3.1 \mathrm{keV}$ оптимальным значением смещения точки фокусировки относительно верхнего края сопла является положение $L_{z}=2 \mathrm{~mm}$. Отметим, что падение выхода рентгена при удалении от края сопла связано с уменьшением плотности газовой струи, а при слишком сильном приближении к краю обусловлено, по-видимому, ростом затрат энергии на генерацию быстрых частиц.

Зависимость числа рентгеновских фотонов от величины входного давления аргоновой струи показана на рис. $2, b$. В отличие от кластерных мишеней, для которых входное давление является критическим параметром, в случае аргоновых газовых струй эмиссия рентгеновского излучения не сильно зависит от входного давления. Начальный рост кривой до давления порядка $6 \mathrm{MPa}$ обусловлен, очевидно, ростом плотности плазмы в области взаимодействия, а дальнейший медленный спад, вероятно, связан с процессами кластеризации. Для используемого в наших экспериментах импульсного сопла оптимальным оказалось давление аргона порядка $6 \mathrm{MPa.}$

Было исследовано также влияние энергии лазерного импульса на нагрев плазмы и соответственно на характер РЭ. Из рис. 3 видно, что изменение энергии лазерного импульса от 50 до $280 \mathrm{~mJ}$ меняет как соотношение спектральных компонент, так и их пиковую интенсивность. При максимальной энергии лазерного импульса $E_{l a s}=280 \mathrm{~mJ}$ в спектрах присутствуют линии Hе-, Liи Ве-подобных ионов аргона, а пиковая спектральная интенсивность (в области линии $\mathrm{He}_{\alpha}$ ) достигает за одну вспышку $\sim 10^{12}$ photons/Å. Постепенное уменьшение энергии лазерного импульса приводит к появлению в спектрах сателлитных линий, отвечающих переходам в B-, C- и N-подобных ионах аргона. Однако при энергии $E_{l a s}=50 \mathrm{~mJ}$ относительная интенсивность сателлитных групп все еще не превышает значения относительной интенсивности резонансной линии $\mathrm{He}_{\alpha}$. При этом пиковая интенсивность линий $\mathrm{He}_{\alpha}$ и группы линий Li-подобных сателлитов снижается примерно на порядок. Эксперименты показали, что число фотонов с энергиями в области 2.9-3.3 keV примерно линейно зависит от энергии лазерного импульса и при $E_{l a s}=280 \mathrm{~mJ}$ составляет $3.5 \cdot 10^{10}$. 
Измеренные рентгеновские спектры были использованы для определения параметров создаваемой плазмы. Для этого проводилось их сравнение с результатами атомно-кинетического моделирования, выполненного в стационарном приближении при помощи радиационно-столкновительного кода PrismSpect [8]. Из рис. 3 видно, что модельные спектры очень хорошо описывали эксперимент при электронных температурах плазмы порядка 190-220 eV.

Большой интерес представляет зависимость выхода рентгена от длительности лазерного импульса. К сожалению, плавное варьирование длительности импульса в широких пределах в рамках одной лазерной установки обычно невозможно. В настоящей работе мы смогли сравнить только режимы нагрева при длительностях 40 и $100 \mathrm{fs}$. Эксперименты показали, что при увеличении длительности лазерного импульса в этих пределах наблюдается интенсивный рост спектральных компонент, отвечающих переходам в Не- и Li-подобных ионах apгона. Эмиссия рентгеновского излучения при этом возрастает примерно на $40 \%$, а число фотонов с энергиями $E_{X-r a y} \sim 3.1( \pm 0.2) \mathrm{keV}$, высвечиваемых за одну лазерную вспышку в полный телесный угол, достигает величины $\sim 7 \cdot 10^{10}$ фотонов, что более чем на порядок превышает значение, зарегистрированное ранее в $[1,2]$.

\section{Финансирование работы}

Работа поддержана грантом Российского фонда фундаментальных исследований 18-52-53033 ГФЕН_а.

\section{Конфликт интересов}

Авторы заявляют, что у них нет конфликта интересов.

\section{Список литературы}

[1] Rousse A., Phuoc T.K., Shah R., Pukhov A., Lefebvre E., Malka V., Kiselev S., Burgy F., Rousseau J.P., Umstadter D., Hulin D. // Phys. Rev. Lett. 2004. V. 93. N 13. P. 135005 (1-4).

[2] Chen L.M., Yan W.C., Li D.Z., Hu Z.D., Zhang L., Wang W.M., Hafz N., Mao J.Y., Huang K., Ma Y., Zhao J.R., Ma J.L., Li Y.T., Lu X., Sheng Z.M., Wei Z.Y., Gao J., Zhang J. // Sci. Rep. 2013. V. 3. P. 1912 (1-5).

[3] Popmintchev T., Chen M-C., Popmintchev D., Arpin P., Brown S., Ališauskas S., Andriukaitis G., Balčiunas T., Mc̈ke O.D., Pugzlys A., Baltuška A., Shim B., Schrauth S., Gaeta A., Hernández-García C., Plaja L., Becker A., JaronBecker A., Murnanel M., Kapteyn H.C. // Science. 2012. V. 336. N 6086. P. 1287-1291.

[4] Purvis M.A., Shlyaptsev V.N., Hollinger R., Bargsten C., Pukhov A., Prieto A., Wang Y., Luther B.M., Yin L., Wang Sh., Rocca J.J. // Nature Photon. 2013. V. 7. N 10. P. 796-800.

[5] Sedov M.V., Faenov A.Ya., Andreev A.A., Skobelev I.Yu., Ryazantsev S.N., Pikuz T.A., Durey P., Doehl L., Farley D., Baird C.D., Lancaster K.L., Murphy C.D., Booth N., Spindloe C., Platonov K.Yu., McKenna P., Kodama R., Woolsey N., Pikuz S.A. // Laser Part. Beams. 2019. V. 37. N 2. P. $176-183$.
[6] Wang J., Feng J., Zhu Ch., Li Y., He Y., Li D., Tan J., Ma J., Chen J. // Plasma Phys. Control. Fusion. 2018. V. 60. N 3. P. 034004.

[7] Alkhimova M.A., Skobelev I.Yu., Faenov A.Ya., Arich D.A., Pikuz T.A., Pikuz S.A. // Quant. Electron. 2018. V. 48. N 8. P. 749-758.

[8] Prism Computational Sciences. Software tools for scientific research and commercial applications in the physical sciences and engineering. http://www.prism-cs.com 